\title{
Smart Tutor an Intelligent Tutoring System for C Sharp Programming
}

\author{
Asma Khan \\ Bahria University Karachi Campus \\ M. Junaid Quadri \\ Muhammad Noman \\ Bahria University Karachi Campus Bahria University Karachi Campus
}

\begin{abstract}
In this paper, application software has been presented called as smart Tutor, which in reality an intelligent tutoring System. The proposed system will let the user to learn the C\# programming language. The decision making process conducted in our intelligent system is guided by a Bayesian network approach to support students in learning computer programming, which is framework for uncertainty management in the field of Artificial management. This system would provide a platform for the students to understand the basic programming concepts through sequence of training concept of programming language. In this paper we will discuss about how system is integrated with Bayesian network as an inference engine to improve student learning process. The significance of this work is that it has replaced the traditional Static Tutorials, Where students learn through video tutorials or text lectures who never come to know about the student learning potential or either the learner has learnt the topic properly before moving on to the next topic. ST will lead the student to navigate through all the available online Course material and will provide the necessary recommendations.
\end{abstract}

\section{Keywords}

Intelligent Tutoring System, Bayesian Network

\section{INTRODUCTION}

It's a time when one use to prefer web based online Tutorials over Traditional text book or "just-put-it-on-the-web" approach in web based system [7], as web based courses offer greater flexibility and are easy to learn anything over internet but the traditional web based learning systems are HTML based web pages and are fail to provide adaptively and interactivity for the learner and are unable to satisfy the heterogeneous needs of today's learner.

Proposed system, that provide you with suggestions and a proper reading sequence, For example a student wants to learn a "for loop" in C\# without learning prerequisite of the required material then our system can determine prerequisite of "for loop" like variable initialization, condition and variable updates and display links for these concept in proper learning sequence to understand the computer programming language.

In this way, one can address the problem of Web-based learners' unproductive navigation, and refocus them on their study objectives by making the tutoring systems adaptable to different types of learners. Studies have showed that one-onone tutoring is the most effective and adaptive mode of teaching and learning. On the Back end the Bayesian Network will carry out the decision process and to get manage with uncertainty management on the basis of student learning Outcome.

We believe that proposed application will be useful for institutions who offer computer programming in their institutions. In particular, this research work is important for institutions where it is difficult to provide personalized instruction that they need. Moreover, we believe that proposed application is very useful to any work applying Bayesian networks as a model for developing adaptive Web-based education tools for different courses.

The reading sequence will be guided by Bayesian network on the basis of provided knowledge to the proposed system. Bayesian networks have been chosen by many web intelligence researchers to perform many tasks, including student monitoring, e-commerce and multi-agents.

\section{INTELLIGENT TUTORING}

Many Computer Assisted Instructional systems have been developed by researchers. The purpose of using computers in assisting instruction is to help out students learn the concepts more efficiently and easily. Computer Assisted Instruction (CAI) systems are traditional education systems, who can teach through computers. In such kind of systems instructional materials is presented in a form of rigid tree structure ,to guide the students from one content page to another depending on their responses in the form of answers. The drawback and restriction with such kind of traditional CAI systems is that students' knowledge states and their needs in terms of concepts are not considerable even though such systems are effective and easy to use by learners. Such systems do not generate flexible instructional plans. Instead, fixed and prescribed plan has been followed.

Intelligent Tutoring Systems (ITS) are adaptive instructional systems developed with the application of AI techniques. [9] mentions that ITSs are developed to resemble the process of the one-to-one learning process between the teacher and a student. ITS's have to represent the content to be thought, implement the instructional strategy and provide mechanism for understanding what the student does and does not know. [10] These features can be summarised with the following components: the expertise module evaluating the student's performance and generating instructional contents, the student-modelling module assessing the student's current state and determining his conceptions and reasoning strategies, and the tutoring module selecting instructional material and presenting it

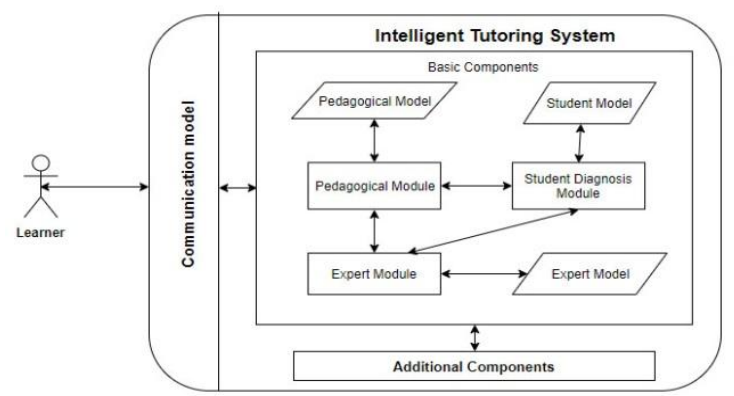

Figure 1: Component of Intelligent Tutoring System 
The key aspect of Intelligent Tutoring System is the student Model. CAI systems are not adaptive and unable to dynamically provide the same kind of individualized attention that students would receive from human teachers. It was a time to indulge Artificial Intelligence techniques in teaching and learning to bring intelligence in CAI systems. The ITS presents is just like one to one tutoring and providing instructions and receiving student feedback. ITS believes that each student is distinctive and unique. The ITS systems can be utilized to teach any kind of course in a more meaningful ,flexible and personalized way and transfer knowledge with the use of internet and keep track of each student learning capability and knowledge during the study. It is comprised of three main tasks. It diagnoses student knowledge level accurately using defined rules of ITS.

Bayesian network will decide what should be the next step and adapt instructions and provide feedbacks.

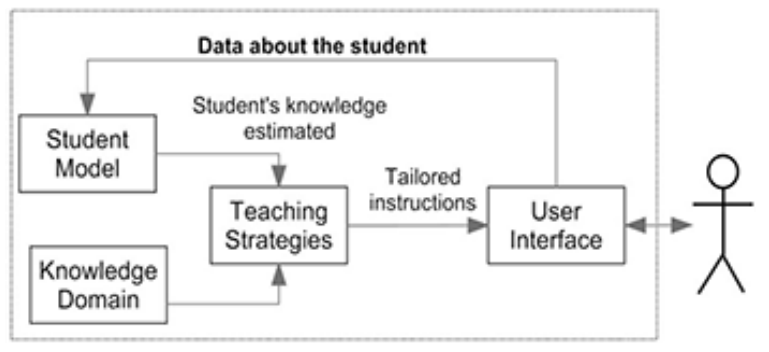

Figure 2: Basic Architecture of an ITS

\section{MAIN COMPONENTS OF SMART TUTOR}

As it has shown in figure 2 that ITS has four components.

\section{a. Knowledge Domain}

It stores all learning material that every student will go through according to the curriculum being taught.

\section{b. Student Model}

This model is responsible to store each student's information and let the system to identify each individual learner and his current knowledge state.

\section{c. Teaching Strategies}

Teaching strategy is very important factor in a sense that way of teaching has great influence on the learner. If teaching strategy is good than it would be easy for learner to grasp the idea and concept. In ITS teaching strategy defines when to show the new topic to learner and how to provide guidance and recommendations for the new topic and quiz. Students assessment result would be provided as an input to this component so the ITS pedagogical decision would define the student needs. At different times, particular teaching methods according to student knowledge level and problems would be defined by this component.

\section{d. User Interface}

This component is responsible to define the systems interactions with the user. So user interface should be well designed to cope up with the needs of ITS and learner.

\subsection{User types}

This will be useful for three type of user:

- Regular Study (Regular registered user)

- Quick Study (Non-registered user)
- $\quad$ Problem Study (Registered user but non-sequential learning)

\subsection{Methodology}

In proposed system, only basic concepts of "C\#" programming language has been covered and common framework of ITS has been implemented and it is comprised of four main components are Bayesian network, the user interface, guidance module and knowledge domain.

System divides user interface in two modules input and output.

\subsection{User Interface}

This section has been divided into two sub modules, input and output.

\section{- Input}

Student should provide the answers to the questions given in quiz and he is responsible to choose his learning goals and specify or state which topics are known to him.

\section{- Output}

As far as output module is concerned, it will show lecture notes to students and will present sample quizzes related to each topic appropriately and will recommend new topics to student according to his knowledge level.

\subsection{Knowledge Domain}

All the lecture notes comprised of basic concepts of C sharp belong to knowledge domain. The knowledge base has Web pages containing class lecture material, All the class tests and their solutions are presented in the form of web pages. After the student has planned to learn a new topic using provided lecture notes, at this point the proposed system will decide about to show the sample quiz related to that particular topic, which are organized by knowledge concepts for C\# programming .Using this approach, we separate knowledge concepts from actual instructional contents, and this separation is advantageous in many ways.

\subsection{Guidance Model}

Guidance module is comprised of three parts. Navigation menu, Prerequisite Recommendations and Learning Sequences. The student model is greatly influenced by the Bayesian network approach.

\section{- Navigation Menu}

Navigational menu is the custom menu that produce for each user through its log provide with list of concept marked with three signs

$$
\begin{array}{ll}
\text { 1. } & \text { Learn } \\
\text { 2. } & \text { Not learn } \\
\text { 3. } & \text { Ready to learn }
\end{array}
$$

Learn and not learn are marked as per user log but the ready to learn is marked by analyzing every learn concept and concept whose prerequisite is the concept learned by the user and by the statistics of asked concept on the basis of prerequisite concept statistics.

Smart tutor treat every user as a unique entity and store every step as a log so to give proper navigational menu for user.

\section{- Prerequisite Recommendations}

As the idea for the proposed system is to provide proper learning, for this it is desired for user to learn all prerequisite 
of the concept. If the user asked for the concept whose prerequisite hasn't been learned then it will be asked form user as to learn the prerequisite of the concept with the statistics of the knowledge percentage for asked concept without its prerequisite, but this won't be a forced act of the system if user ignore the recommendations.

\section{- Learning Sequences}

Learning sequence is the main aspect in learning any programming language from basic to have proper knowledge over the language. For this purpose the proposed system exhibit learning sequence of programming language through many programming prospectus and recommendations from multiple professors. Registered user are asked to use proper sequence provided by the system. Example:

If user registered to the system then the first concept system asked him will be the overview of programming and Clanguage as in Bayesian network nodes figure (in methodology).

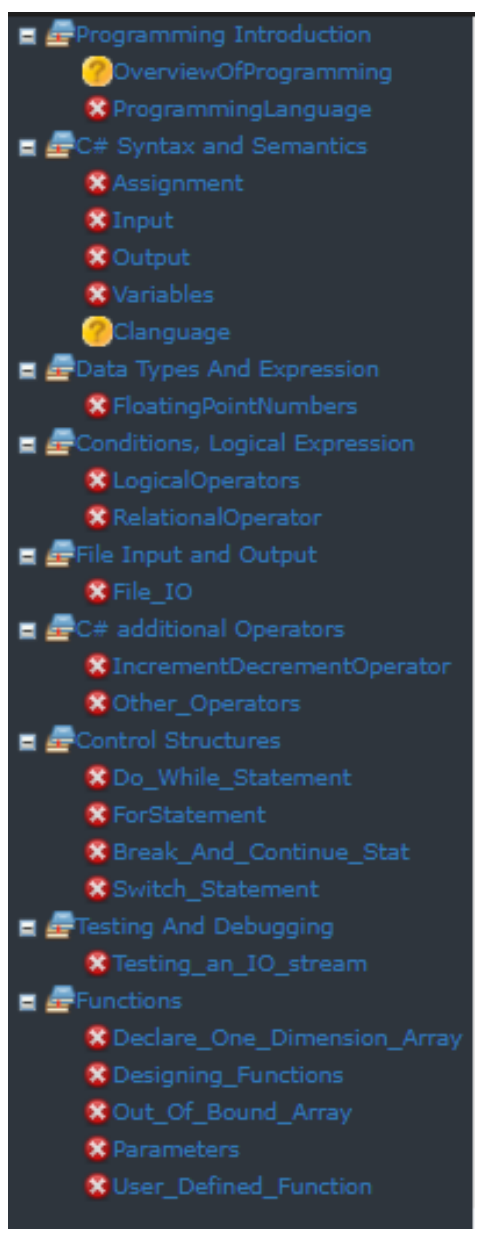

Figure 2: Student Navigation Menu

Following figure showing subjects user can start with in regular study mode. And if user select "Quick study" mode then user will be asked to select required concept and on selection create suggestion on behalf of it.

\section{BAYESIAN NETWORK}

Bayesian network is a representation of nodes(Random variables) and their conditional dependencies via a directed acyclic graph, see Example, showing that factors for wet grass, can be from sprinkle or rain. Each node represents an activity or instance for its derived node to be happen and is a part of it to come.

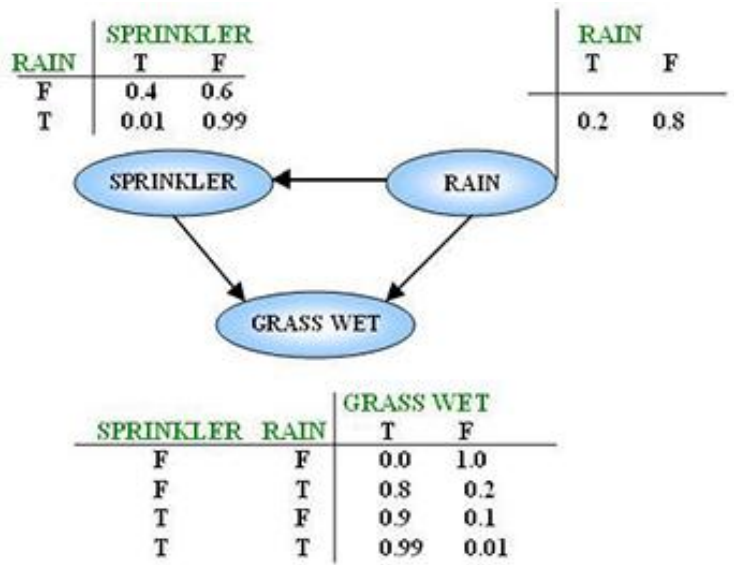

Figure 3: Wet Grass Example

Another example for Medical diagnosis:

We might ask what factors affect a patient's chance of having cancer. If the answer is "Pollution and smoking," then we should add arcs from Pollution and Smoker to Cancer. Similarly, having cancer will affect the patient's breathing and the chances of having a positive X-ray result. So we add arcs from Cancer to Dyspnea and X-ray. The resultant structure is shown in Figure 1. It is important to note that this is just one possible structure for the problem

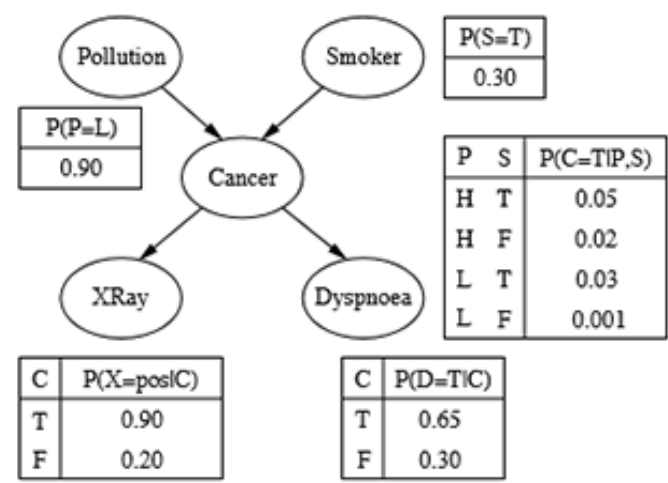

Figure 4: Lung Cancer Example

Main reason for using Bayesian Network in "Tutoring System "is the student uncertainty management, which means to reduce certain possible reasons for student to get proper required knowledge, which is in our system is " $\mathrm{C \#}$ Programming Language(Basics)”.

\section{- Bayesian Network in Intelligent tutoring} System:

1. First to create a network that's satisfy basic programming language curriculum so to have a proper connectivity of nodes(concepts in our system).

I. Multiple software are freeware to build Bayesian network and do reasoning, prediction \& diagnostics like "NETICA" and "MSBNx", this system uses MSBNx for building the required network

II. Resulting in network (At the end of paper)

III. MSBNx comes with functionality to define probabilities within each node, show figure: 


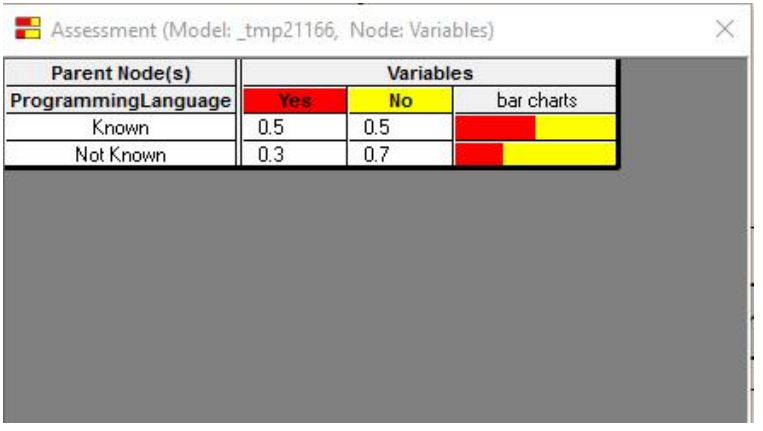

Figure 5: Node Assessment representation in MSBNx

The row identifier known and not known are the conditions to check if a person known / not known with the concept (Programming Language defined in the figure) then the probability of getting the parent concept is defined in the table where yes means the person will get this concept with this percent of knowledge understanding and no for not getting this percent of knowledge about the concept.

IV. Now what to fill in the probability tables, see Example:

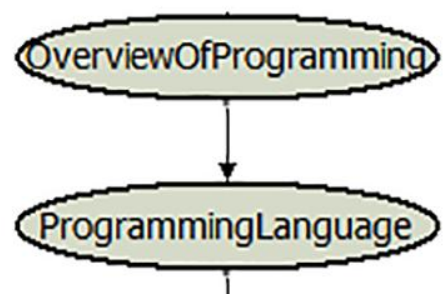

Figure 6 : Node Relation with Parent Node MSBNx

Data: (which is in this case is dummy data)

Overview Of Programming $=O P$

ProgrammingLanguage $=P L$

$$
\begin{aligned}
& P(O P=\text { true })=0.7 \\
& P(O P=\text { false })=0.3 \\
& P(P L=\text { true } / O P=\text { true })=0.8 \\
& P(P L=\text { true } / O P=\text { false })=0.4
\end{aligned}
$$

So, if a user comes to system with not the knowledge about the "Overview of Programming" then the possibility of getting Programming language will be above percentage specified.

V. Now to fill all the conditional probability tables, we need to gather all the aspect from the Bayesian network and all its condition. For example a condition that what is the probability of a student to know about concept (for loop) for if that person knows about the prerequisite of it.

\section{Now how the system create this kind of actions}

Firstly if new users come to the system, then they should be asked to register themselves for the system. Now if the user registers then a new user instance created in the database, with their recent activity as nothing so the system recognized him as a new user so to provide with introductory operations.
Now for learning purpose the new user will be provided with required course for user. Then user register himself and after registering the user will be provided with the curriculum sequence and lecture showing in a sequential manner. After each ending tutorial or curriculum concept the user is asked for concept to be known by him or not or some confusions, then the system will provide the quiz so to check if the user gain knowledge about the concept, for quiz will provide the percentage of the knowledge user gets. If it satisfies to go further then user will be provided with the next concept.

Now there are users that want frequent knowledge about the concept rather than going with the sequential concept to get to the required ones. To cater this problem each user recent activity and the log created each time it comes to learn a concept either frequent or regular learner.

So logs in this system is student records, records to remember each student knowledge level and make system acts like a teacher giving you a whole semester course. Now the logs are the main memory of each student, so it is necessary to make acknowledgement about the students understanding of the recent topic studying to store in. Smart tutor create logs after every completion of topic with giving three choices asking:

1. Have you get the concept

2. I am not sure, quiz will be sufficient to make it properly understand

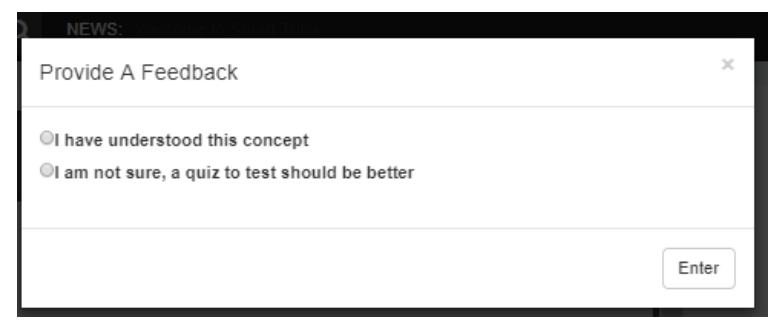

Figure 7 : System Gathering FeedBack from Students

Taking First choice from the above will make a user log in the system providing user with the further topic.

And for the $2^{\text {nd }}$ one, system will provide a quiz to the user that will outcome the user understanding of the concept in the form of percentage of acknowledgement for condition to decide if user gets the knowledge or not to give further detailed version of the concept or promoting to next concept. 


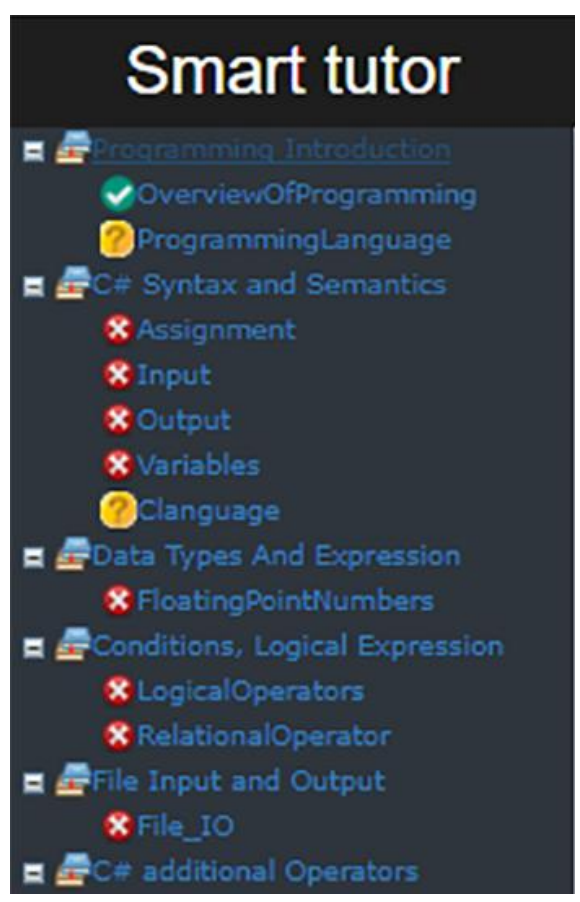

Figure 8 : Navigational Menu for Quick Mode Users

For Quick mode users, Smart tutor will specify what subject they have learned through the website, because every activity of a user is logged in smart tutor.

1. Modeling the Problem Domain.

Bayesian networks will be using in the proposed project to achieve the following objectives ; firstly student will be helping out to navigate through the web based learning system so, problem domain must be modeled ;secondly proposed will keep track of previous knowledge of student regarding each concept in the problem domain. In proposed intelligent tutoring system, the scope of the problem is restricted Initially, the system is built to teach students C\# programming language, and only elementary topics are covered, which can be found in introductory courses on programming. These concepts include concepts such as variables, assignments, and control structures (while, Do while and For Loop Control Structures), while more complex topics like pointers and object Oriented concepts are excluded and can be considered for future work.

Bayesian Network, will define the prerequisite relationships among the topics and directly and clearly.

In order to find out either student has learned the concept properly or not, a sample quiz of corresponding topic will be presented to student, and quiz result will determine, student has understood the topic properly or he needs to read the topic again or he can move to learn the new concept. Although a quiz question may pertain to several C\# topics, we subjectively associated each question with the most relevant topic.

System presents better method of estimation and will estimate the student level of knowledge in terms of quiz results. After the student finishes reading the displayed lecture notes, will acknowledge with feedback about concept. System added a $\log$ for that user regarding particular topic so to create proper action for further. More specifically, he or she selects from one of the following three choices: I understand this concept; I don't understand this concept; I'm not sure (quiz me). These can be asked from user.

\section{RESULTING BAYESIAN NETWORK}

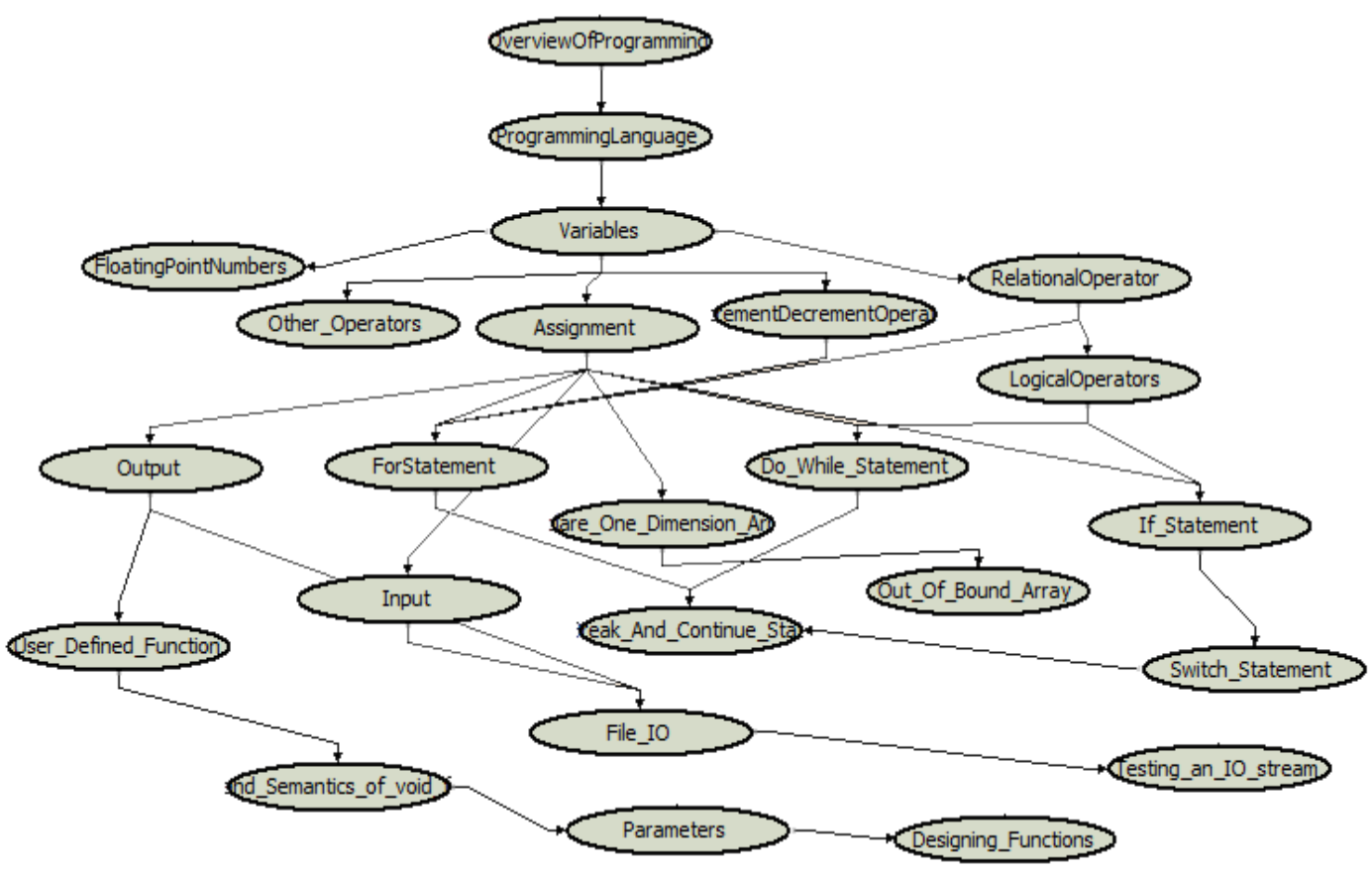

Figure 9: Bayesian Network Implemented in Smart Tutor 


\section{CONCLUSIONS}

In this paper, we proposed an Intelligent tutoring system (Smart Tutor) for computer programming that uses bayesian network, A concept to manage uncertainty which is in our case is programming guidance to user on the basis of hypothesis provided to the bayesian network in our system. As in every aspect artificial intelligence is taking place, Using Bayesian network which is a good way to removing uncertainty management to provide a way of tutoring system that will look every user as a single one to provide a one to one learning environment. Smart tutor take each student as a unique user and track user activity about learning concept and $\log$ it looking forward to give proper sequence and proper suggestion about the prerequisite concept for chosen one through learning sequence and alerts.

Firstly Currently most web based learning systems are simple websites that give only the text based lecture or videos, other than that if a user appear to have no knowledge about the concept won' t know what should learn first or after, the proposed system is an approach to provide complete knowledge about $\mathrm{C} \#$ programming to a person not even familiar with programming using proper sequence, which is the most important aspect a teacher make effort to provide knowledge over the semester to the class, the sequence the system uses is generated through people related to teaching in university. Secondly most web based learning system didn't create suggestion on the basis of previous record of user as they didn't save any. The proposed system is functioned to create suggestion on the basis of logs created every time user learned a concept and system make an acknowledgement for user and get info about user knowledge about the given concept and create a log for it, so next time user login will proceed to next step on the other hand these acknowledgement are useful for system to create learning sequence, suggestions and alerts to user for prerequisites.

This system is also designed to take quiz for any concept if user acknowledges about the quiz that is one of the acknowledgment system ask from users. These quiz result are useful for system as to decide the probability of user knowledge gained over the concept for suggestion to go further from the sequence or review the previous one.

The proposed system can be used with any other language but with only the concept defined on the network.

\section{FUTURE WORK}

Smart tutor will be used for further advanced concepts that are next level of the course other than the one given on the network. Data will be collected for these further concepts and add to the network.
The proposed system is using classification for user from data specified on the Bayesian network, Smart tutor will be used to learn from user as the quiz system is the key aspect that will be used for collected data from user and specify on the Bayesian network to increase correctness possibility.

\section{REFERENCES}

[1] A Bayes Net Toolkit for Student Modeling in Intelligent Tutoring Systems Kai-min Chang, Joseph Beck, Jack Mostow, and Albert Corbett

[2] Uncertainty Management for Diagnostics and Prognostics of Batteries using Bayesian Techniques.

[3] "A Brief Introduction to Graphical Models and Bayesian Networks", By Kevin Murphy, 1998.

[4] Mitrovic, A. (2003). An Intelligent SQL Tutor on the Web. International Journal of Artificial Intelligence in Education, 13(2-4), 171-195

[5] Using Bayesian Networks to Manage Uncertainty in Student Modeling, by Cristina Conati, Abigail Gertner and Kurt VanLehn

[6] A Web-based Bayesian Intelligent Tutoring System for Computer Programming, C.J. Butz, S. Hua, R.B. Maguire Department of Computer Science

[7] Adaptive and Intelligent Web-based Educational Systems, Peter Brusilovsky and Christoph Peylo

[8] The Past, the Present and the Future of adaptive ELearning, Felix Mödritscher, Victor Manuel GarciaBarrios and Christian Gütl.

[9] Shute, V.J.; Psotka, J.: Intelligent tutoring systems: Past, present and future. In book Handbook of research on educational communications and technology, 1995.

[10] Advances in Intelligent Tutoring Systems: Problemsolving Modes and Model of Hints, Alla AnohinaNaumeca

[11] C.W. Woo, Instructional Planning in an Intelligent Tutoring System: Combining Global Lesson Plans with Local Discourse Control, Ph.D. Thesis, Illinois Institute of Technology, Chicago, IL, 1991

[12] N.L. Zhang, D. Poole, A simple approach to Bayesian network computations, in: Proceedings of The 10th canadian Conference on Artificial Intelligence, Banff, Alberta, Canada, 1994, pp. 171-178

[13] Jim Reye. Student Modelling based on Belief Networks. International Journal of Artificial Intelligence in Education (IJAIED), 2004, 14, pp.63-96. 